\title{
Characterizations of Modified Silica Nanoparticles(I)
}

\author{
Seong Kee Min”, Chan Young Park, Won Ki Lee and Soo Duk Seul* \\ Department of Polymer Engineering, Pukyong National University, Busan 608-739, Korea \\ *Department of Chemical Engineering, Dong-A University, Busan 604-714, Korea
}

(Received April 18, 2012 : Received in revised form May 19, 2012 : Accepted May 21, 2012)

\begin{abstract}
MPTMS) was used as a silylation agent, and modified silica nanoparticles were prepared by solution polymerization. $2.0 \mathrm{~g}$ of silica nanoparticles, $150 \mathrm{ml}$ of toluene, and $20 \mathrm{ml}$ of MPTMS were put into a $300 \mathrm{ml}$ flask, and these mixtures were dispersed with ultrasonic vibration for $60 \mathrm{~min} .0 .2 \mathrm{~g}$ of hydroquinone as an inhibitor and 1 to 2 drops of 2,6-dimethylpyridine as a catalyst were added into the mixture. The mixture was then stirred with a magnetic stirrer for $8 \mathrm{hrs}$. at room temperature. After the reaction, the mixture was centrifuged for $1 \mathrm{hr}$. at $6000 \mathrm{rpm}$. After precipitation, $150 \mathrm{ml}$ of ethanol was added, and ultrasonic vibration was applied for $30 \mathrm{~min}$. After the ultrasonic vibration, centrifugation was carried out again for $1 \mathrm{hr}$. at 6000rpm. Organo-modification of silica nanoparticles with a $\gamma$-methacryloxypropyl functional group was successfully achieved by solution polymerization in the ethanol solution. The characteristics of the $\gamma$-mercaptopropyl modified silica nanoparticles (MPSN) were examined using X-ray photoelectron spectroscopy (XPS, THERMO VG SCIENTIFIC, MultiLab 2000), a laser scattering system (LSS, TOPCON Co., GLS-1000), Fourier transform infrared spectroscopy (FTIR, JASCO INTERNATIONL CO., FT/IR-4200), scanning electron microscopy (SEM, HITACHI, S-2400), an elemental analysis (EA, Elementar, Vario macro/micro) and a thermogravimetric analysis (TGA, Perkin Elmer, TGA 7, Pyris 1). From the analysis results, the content of the methacryloxypropyl group was $0.98 \mathrm{mmol} / \mathrm{g}$ and the conversion rate of acrylamide monomer was $93 \%$. SEM analysis results showed that the organo-modification of ultra-fine particles effectively prevented their agglomeration and improved their dispensability.
\end{abstract}

Key words $\mathrm{SiO}_{X}$, SAMs, silylation, nanoparticle, dispensability.

\section{Introduction}

Many different methods have recently been attempted to carry out the research that controls the characteristic of a solid surface with organic matter. One of them is selfassembled monolayers (SAMs). ${ }^{1-3)}$ There is a way to selfassemble what is linked with silanol groups $(-\mathrm{SiOH})$ of monolayers of organosilicon derivatives and $\mathrm{Si}-\mathrm{O}-\mathrm{Si}$ coherence fixes with a covalent bond as a method to fix selfassembled monolayers to inorganic supports with a covalent bond and a method to produce polysiloxane etc. ${ }^{4)}$

The silylation agents are organosilicon compounds to substitute silicone atoms for active hydrogen of the organic compounds. They are mainly used to protect functional groups containing the active hydrogen when polymerize ingredients or medium in the field of medical supplies. Besides, they are used to reform silicon wafers or glass plates surface in the field of electronics. It is expected that the silylation agents can serve as the process chemical considering the environment, economical efficiency, and

${ }^{\dagger}$ Corresponding author

E-Mail : minsk@pknu.ac.kr (S. K. Min) energy saving. ${ }^{5-8)}$

The functional silane with surface variation of nanoparticles is one of the important methods, ${ }^{9-11)}$ because this is able to change the surface characteristic of ultra-fine particles and protect the particles from condensation process. ${ }^{12)}$ Moreover, it can be possible that this inducts 'reacting site' onto the surface of silica nanoparticles. Among a wide range of matrices, including clay mineral, oxides, and glasses, what is able to contain the $\mathrm{OH}$ end groups bonded on surface, the silica gel is changed to a variety of alkoxysilane with other functionality. So, this can not only obtain silica nanoparticles with different surface chemicals and physical performance with ease, but is also likely to play an important role in the material industries (e.g. additive of lubricating oil and organic-inorganic hybrid materials).

In this study, (3-mercaptopropyl)trimethoxysilane (MPTMS) was used as silylation agents, and modified silica nanoparticles were prepared by the self-assembly method. The characteristics of MPSN were examined using X-ray photoelectron spectroscopy (XPS), laser scattering system (LSS), Fourier transform infrared spectroscopy (FTIR), scanning electron microscope (SEM), elemental analysis (EA) and thermo gravimetric analysis (TGA). 
Table 1. The properties of silica nanoparticles.

\begin{tabular}{lcc}
\hline \multicolumn{1}{c}{ Properties } & Unit & $\begin{array}{c}\text { Typical } \\
\text { value }\end{array}$ \\
\hline $\mathrm{SiO}_{2}$ content (based on ignited material) & $\mathrm{wt} \%$ & $\geq 99.8$ \\
specific surface area $(\mathrm{BET})$ & $\mathrm{m}^{2} / \mathrm{g}$ & $300 \pm 30$ \\
average primary particle size & $\mathrm{nm}$ & 7 \\
moisture $\left(2\right.$ hours at $\left.105^{\circ} \mathrm{C}\right)$ & $\mathrm{wt} \%$ & $\leq 3$ \\
ignition loss $\left(2\right.$ hours at $\left.950^{\circ} \mathrm{C}\right)$ & $\mathrm{wt} \%$ & $\leq 2$ \\
$\mathrm{pH}(5 \mathrm{wt} \%$ in water) & & $3.7 \sim 4.7$ \\
\hline
\end{tabular}

\section{Experimental Procedure}

\subsection{Materials and reagents}

The silica nanoparticles are products of Nanotech ceramics and their characteristics are shown in Table 1. These characteristics were provided by Nanotech ceramics.

This silica was dried in vacuum at $110^{\circ} \mathrm{C}$ for $48 \mathrm{hrs}$. before experiment. (3-mercaptopropyl)trimethoxysilane (MPTMS) is a reagent of Aldrich and its degree of purity is above 95\%. 2,6-Dimethylpyridine (Junsei), toluene (Junsei), hydroquinone (Kanto), and ethanol (SK) were used as the catalyst, solution, antipolymerization and reaction extraction solution, respectively.

\subsection{Methods}

\subsubsection{Self-assembly procedures}

$2.0 \mathrm{~g}$ silica nanoparticles, $150 \mathrm{ml}$ toluene and $20 \mathrm{ml}$ (3mercaptopropyl) trimethoxysilane (MPTMS) were put into a $300 \mathrm{ml}$ flask, and these mixtures are dispersed with ultrasonic vibration for $60 \mathrm{~min}$. $0.2 \mathrm{~g}$ hydroquinone as the inhibitor and 1 to 2 drops of 2,6-dimethylpyridine as the catalyst were added into the mixture. Then, the mixture should be stirred with magnetic stirrer for $8 \mathrm{hrs}$. at room temperature. After the reaction, the mixture is centrifuged for $1 \mathrm{hr}$. at 6000rpm. After the precipitation, adding 150 $\mathrm{ml}$ ethanol, giving ultrasonic vibrations for $30 \mathrm{~min}$. After the ultrasonic vibrations, the centrifuge is tried again for $1 \mathrm{hr}$. at 6000rpm. $\gamma$-mercaptopropyl modified silica nanoparticles (MPSN) results from three-time-repetition of this process and drying process in vacuum at room temperature.

\section{Results and Discussion}

\subsection{Elemental analysis}

In this study, the amount of anchored functionality of MPSN was $0.98 \mathrm{mmol} / \mathrm{g}$ obtained from the results of C elemental analysis. The methoxyl group was perfectly hydrolyzed and adhered to silica nanoparticles by covalent bond.

Elemental analysis results of bare $\mathrm{SiO}_{X}$ nanoparticles and MPSN were shown in Table 2. From analysis results,
Table 2. Elemental analysis.

\begin{tabular}{ccc}
\hline Contents $(\%)$ & bare silica & MPSN \\
\hline $\mathrm{C}$ & - & 5.29 \\
$\mathrm{H}$ & - & 1.14 \\
$\mathrm{~N}$ & - & 4.75 \\
\hline
\end{tabular}

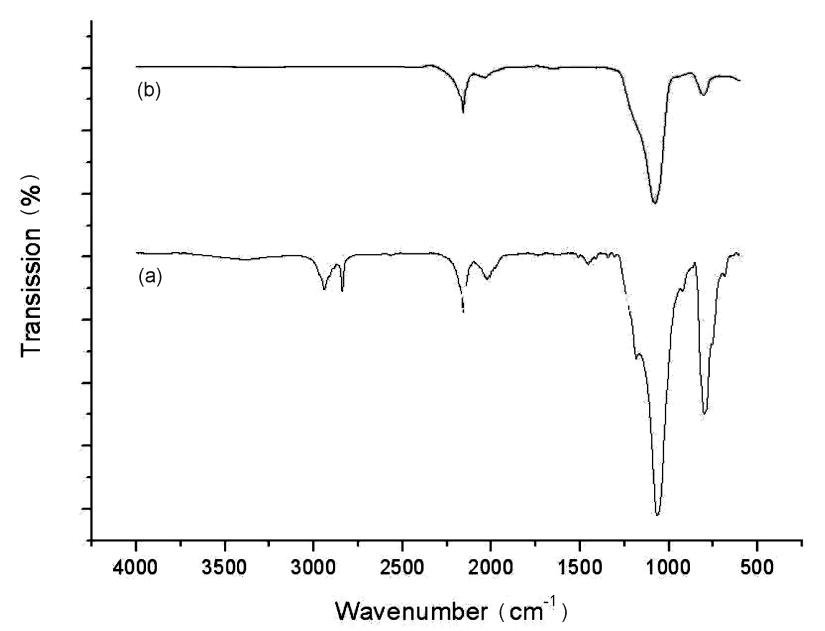

Fig. 1. Infrared spectra of (a) the bare silica nanoparticles and (b) MPSN.

the content of methacryloxypropyl group is $0.98 \mathrm{mmol} / \mathrm{g}$ and the conversion rate of acrylamide monomer is $93 \%$.

\subsection{FT-IR analysis}

Infrared spectroscopy was used as an instrument to provide information on the total or partial loss of silanol groups bonded to the original silica nanoparticles, and to clarify the effect caused by the immobilization of organic group on the surface of organo-modified silica nanoparticles. In Fig. 1, Si-O-Si was observed at $1079 \mathrm{~cm}^{-1}$. On the surface of silica, terminal Si-OH deformation and $\delta$ Si$\mathrm{O}$ occurred at $959 \mathrm{~cm}^{-1}$ and $464 \mathrm{~cm}^{-1} \cdot\left(\mathrm{CH}_{2}\right)_{3}$ group, $\mathrm{C}=\mathrm{C}$ group and $\mathrm{C}=\mathrm{O}$ group are presented at $1454 \mathrm{~cm}^{-1}, 1627$ $\mathrm{cm}^{-1}$ and $1710 \mathrm{~cm}^{-1}$, respectively. Si-O group located at $801 \mathrm{~cm}^{-1}$ in FTIR results from silica decreasing the peak because of the addition of Si-C in MPSN.

\subsection{XPS analysis}

An X-ray is an electromagnetic radiant light of minor wavelength which is generated from the retardation of high energy electron or the electron transition of atomic internal orbital electron. The XPS spectral shifts in the binding energy of individual peaks can indicate the species present. The ability to use these shifts in binding energy is what makes XPS a valuable analytical technique for industrial applications where bonding information is required.

\subsubsection{Bare silica}

Fig. 2 shows XPS spectrum of bare silica. More specific- 

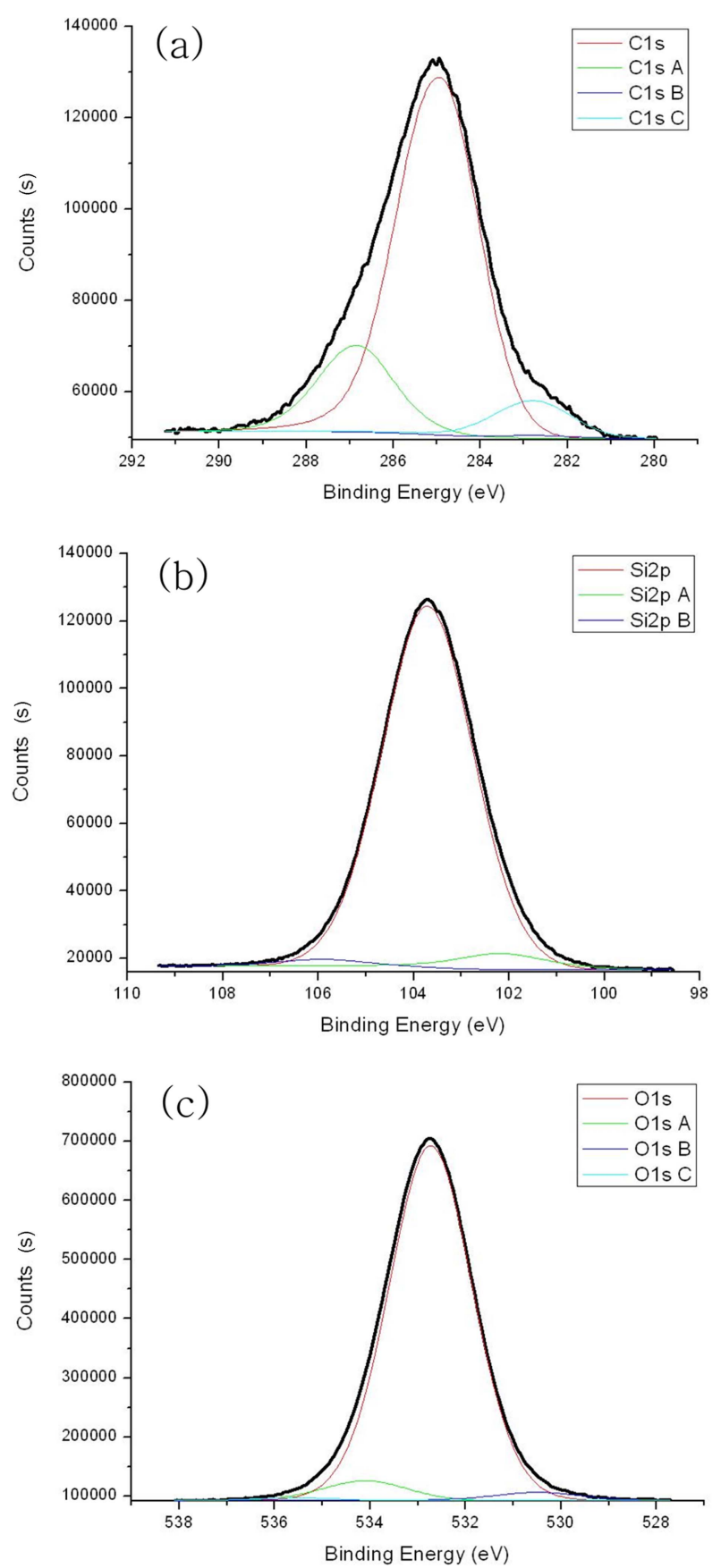

Fig. 2. XPS spectra of bare silica : (a) $\mathrm{C} 1 \mathrm{~s}$, (b) $\mathrm{Si} 2 \mathrm{p}$ and (c) O1s.

ally, Fig. 2(a) shows that $\mathrm{C} 1 \mathrm{~s}$ content peak is presented at $284.93 \mathrm{eV}$, Fig. 2(b) shows that Si2p content peak is presented at $103.70 \mathrm{eV}$, and Fig. 2(c) shows that O1s peak is presented at $532.73 \mathrm{eV}$. When Fig. 2 is compared with Fig. 3, it is possible to presuppose reactivity of silica and silanol groups.

\subsubsection{MPSN}

In Fig. 3, C1s, Si2p and O1s spectra of MPSN are analyzed to investigate the chemical combination condi-
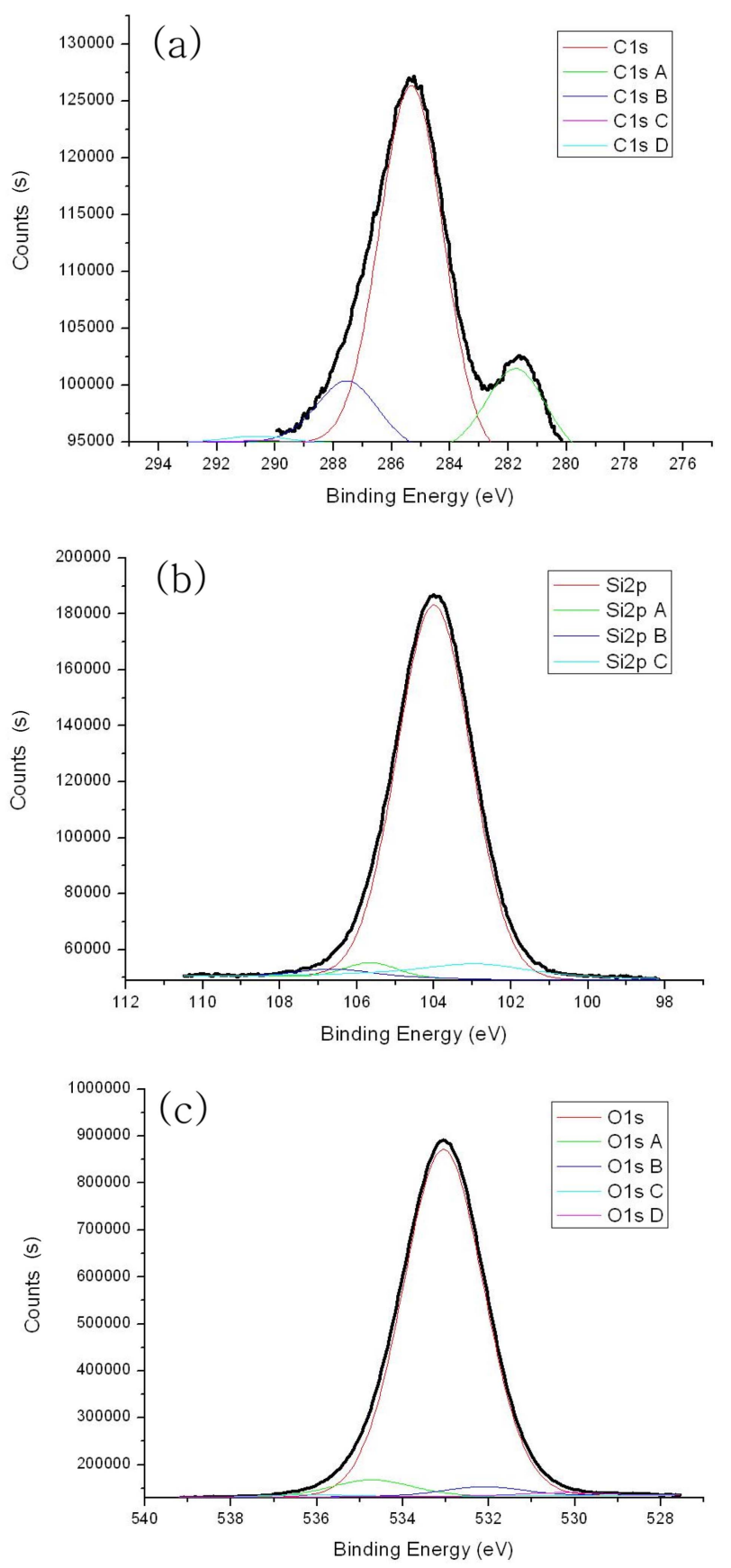

Fig. 3. XPS spectra of MPSN : (a) C1s, (b) Si2p and (c) O1s.

tions of carbon, silicon and oxygen and amount of functionality group determining rate. Fig. 3(a) shows that $\mathrm{C} 1 \mathrm{~s}$ peaks of MPSN are able to resolve into four elements. The first, $281.73 \mathrm{eV}$, is ascribed to carbon in an aliphatic environment such as $\mathrm{C}-\mathrm{H}, \mathrm{C}-\mathrm{C}$ and $\mathrm{C}=\mathrm{C}$. The second and third, set at $290.62 \mathrm{eV}$ and $290.34 \mathrm{eV}$, are attributed to carbon bound to oxygen $((\mathrm{C}=\mathrm{O})-\mathrm{O}-\mathrm{C})$. The fourth, $287.52 \mathrm{eV}$, can be ascribed to $\mathrm{C}-\mathrm{Si}$. The $\mathrm{Si}-\mathrm{O}$ at $102.88 \mathrm{eV}, \mathrm{Si}-\mathrm{C}$ at $105.65 \mathrm{eV}$ and Si-C at $106.64 \mathrm{eV}$ are observed in Fig. 3(b). The $\mathrm{O}-\mathrm{C}, \mathrm{O}=\mathrm{C}, \mathrm{O}-\mathrm{Si}$ and $\mathrm{C}-\mathrm{O}-\mathrm{Si}$ are presented at 
Table 3. The surface compositional data from XPS.

\begin{tabular}{ccccc}
\hline & \multicolumn{4}{c}{ XPS analysis (at\%) } \\
Samples & Si $(2 \mathrm{p})$ & O (1s) & C (1s) & N (1s) \\
\hline \multirow{2}{*}{ bare silica } & 30.91 & 44.70 & 20.77 & - \\
& $(103.37 \mathrm{eV})$ & $(532.45 \mathrm{eV})$ & $(284.75 \mathrm{eV})$ & - \\
MPSN & 35.38 & 52.66 & 11.96 & - \\
& $(110.50 \mathrm{eV})$ & $(533.50 \mathrm{eV})$ & $(286.02 \mathrm{eV})$ & - \\
\hline
\end{tabular}

$530.28 \mathrm{eV}, 532.12 \mathrm{eV}, 534.73 \mathrm{eV}$ and $536.23 \mathrm{eV}$ in Fig. 3(c) respectively. The existence of additional component characteristics of carbon element, silicon atoms bound to carbon, and oxygen atoms bound to carbon represents the propyl methacrylate self-assembled monolayer has been the covalent bond onto the surface of silica nanoparticles. This result matches with FTIR analysis results.

The surface component data of the bare silica and MPSN were summarized at Table 3 with the XPS analysis. The $\mathrm{C}, \mathrm{N}, \mathrm{S}$ and $\mathrm{O}$ contents of bare $\mathrm{SiO}_{X}$ nanoparticle were $20.77 \%, 0,30.91 \%$ and $44.70 \%$, respectively. And, the $\mathrm{C}, \mathrm{N}, \mathrm{S}$ and $\mathrm{O}$ contents of MPSN were $11.96 \%, 0$, $35.38 \%$ and $52.66 \%$, respectively. The surface element contents of $\mathrm{C}$ and $\mathrm{N}$ increase to each $63.44 \%$ and $12.00 \%$, while $\mathrm{Si}$ and $\mathrm{O}$ decrease to each $1.34 \%$ and $23.32 \%$. The $\mathrm{SiO}, \mathrm{C}-\mathrm{O}$ and $\mathrm{O}=\mathrm{C}-\mathrm{O}$ type of O1s, $\mathrm{Si}-\mathrm{C}, \mathrm{C}-\mathrm{H}, \mathrm{C}-\mathrm{C}, \mathrm{C}=\mathrm{C}$, $\mathrm{C}-\mathrm{O}$ and $\mathrm{O}-\mathrm{C}=\mathrm{O}$ type of $\mathrm{C} 1 \mathrm{~s}$ exist to core-level spectra of MPSN.

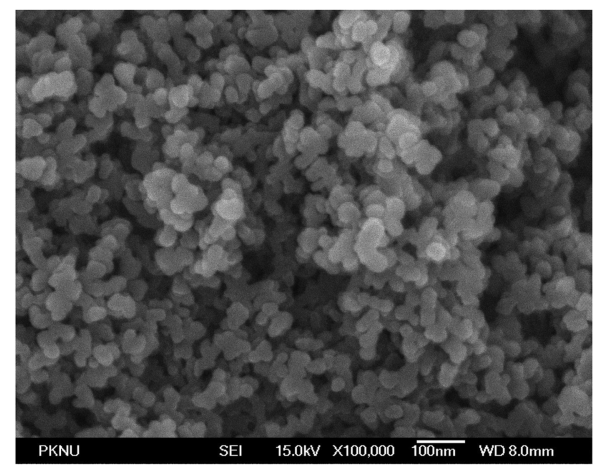

(a)

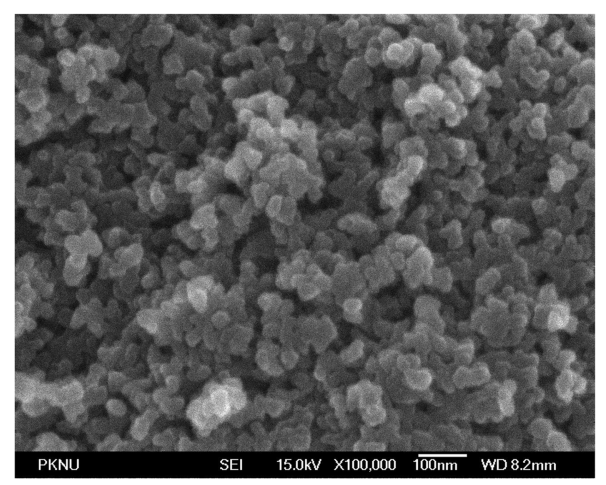

(c)

\subsection{Morphological analysis}

Scanning electron microscopy is used for the morphology analysis of the organo-modified silica nanoparticles with self-assembled monolayers and images of the bare silica nanoparticles. Fig. 4 shows the bare silica and MPSN. In Fig. 4(a), the diameter of bare nanoparticle is about $25 \mathrm{~nm}$. This result is bigger than $7 \mathrm{~nm}$ which is shown in Table 1. This is probably due to the agglomeration at the stocking stage. The organo-modification is able to break up the weak agglomeration of ultra-fine particles and improve their dispensability in organic solvent. This can be seen in Fig. 4(b) and Fig. 4(c). Fig. 4(a) shows the bare silica and Fig. 4(b) shows dispersion of the bare silica with ultrasonic vibration for $30 \mathrm{~min}$. in toluene. Meanwhile, Fig. 4(c) represents dispersion of the bare silica in water. MPSN has a poor dispersibility in ethanol. (See Fig. 4(d)).

\subsection{Thermal analysis}

The thermal stability of MPSN was explored by TGA analysis (Fig. 5). The weight loss of bare silica nanoparticles shows the desorption of some gases absorbed from the air at below $150^{\circ} \mathrm{C}$, but MPSN stabilizes at almost every temperature.

\section{Conclusion}

The organo-modification of silica nanoparticles with $\gamma$ -

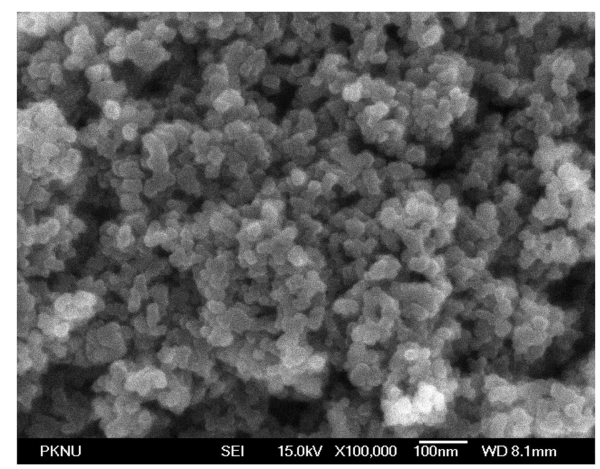

(b)

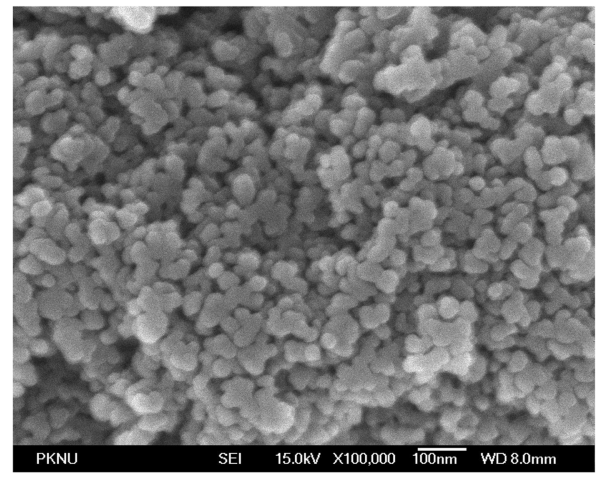

(d)

Fig. 4. SEM images of silica nanoparticles : (a) bare silica, (b) bare silica in tolune 30min, (c) bare silica in water 30min and (d) MPSN. 


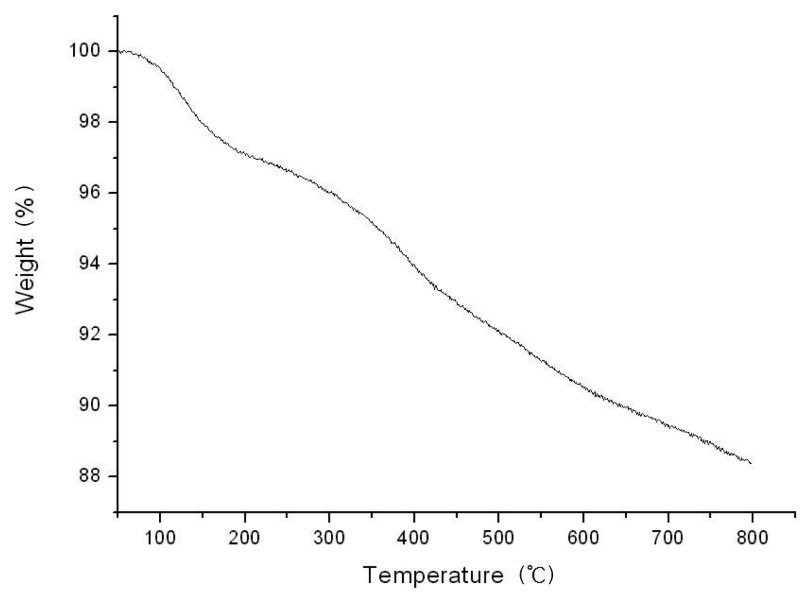

Fig. 5. TGA curve of MPSN.

methacryloxypropyl functional group was successfully prepared by solution polymerization in the ethanol solution. The results of FTIR and XPS analysis showed that the assembled monolayers had been a covalent bond; SEM analysis results showed that the organo-modification of ultra-fine particles were able to prevent their agglomeration effectively and improve their dispensability. It will be used as lubricating oil and organic-inorganic hybrid material in the material industry.

\section{Acknowledgment}

"This work was supported by the Pukyong National University Research Fund in 2010 PK-2010-0012000201016700.”

\section{References}

1. W. C. Bigelow, D. L. Pickett and W. A. Zisman, J. Colloid Sci., 1, 513 (1946).

2. J. Sagiv, J. Am. Chem. Soc., 102, 92 (1980).

3. R. G. Nuzzo and D. L. Allara, J. Am. Chem. Soc., 105, 4481 (1983).

4. A. Ulman, Chem. Rev., 96, 1533 (1996).

5. Y. Haga, S. Inoue, T. Sato and R. Yosomiya, Appl. Macromol. Chem. Phys. Angew. Makromol. Chem., 139(1), 49 (1986).

6. A. G. Mayes and K. Mosbach, Trends Anal. Chem., 16, 321 (1997).

7. B. Sellergren, C. Dauwe and T. Schneider, Macromolecules, 30, 2454 (1997).

8. M. Takenaga, Y. Serizawa, Y. Azechi, A. Ochiai, Y. Kosaka, R. Igarashi and Y. Mizushima, J. Contr. Release, 52, 81 (1998).

9. H. Ni, Y. Du, G. Ma, M. Nagai and S. Omi, Macromolecules, 34, 6577 (2001).

10. H. Ni, G. Ma, M. Nagai and S. Omi, J. Appl. Polym. Sci., 82, 2679 (2001).

11. H. Ni, G. Ma, M. Nagai, and S. Omi, J. Appl. Polym. Sci., 82, 2692 (2001).

12. E. -J. Kim and H. -C. Shin, Kor. J. Mater. Res., 22(1), 8 (2012) (in Korean). 